\title{
Projection de la population des exploitations agricoles françaises à l'horizon 2025 (supplément)
}

\section{Legrand Dunold Fils Saint-Cyr}

\section{(2) OpenEdition}

1 Journals

Édition électronique

URL : http://journals.openedition.org/economierurale/6125

DOI : $10.4000 /$ economierurale. 6125

ISSN : 2105-2581

Éditeur

Société Française d'Économie Rurale (SFER)

\section{Édition imprimée}

Date de publication : 30 septembre 2018

ISSN : 0013-0559

\section{Référence électronique}

Legrand Dunold Fils Saint-Cyr, "Projection de la population des exploitations agricoles françaises à I'horizon 2025 (supplément) », Économie rurale [En ligne], 365 | Juillet-septembre 2018, mis en ligne le 30 septembre 2020, consulté le 05 janvier 2020. URL : http://journals.openedition.org/ economierurale/6125; DOI : 10.4000/economierurale.6125

Ce document a été généré automatiquement le 5 janvier 2020.

(c) Tous droits réservés 


\title{
Projection de la population des exploitations agricoles françaises à l'horizon 2025 (supplément)
}

\author{
Legrand Dunold Fils Saint-Cyr
}

\section{A. Consolidation de la base COTNS à l'échelle des exploitations}

1 Les données de la base des cotisants non-salariés (COTNS) de la Caisse centrale de la mutualité sociale agricole (MSA) portant sur des individus, un travail de consolidation est nécessaire pour pouvoir travailler à l'échelle des exploitations.

La première étape a consisté à sélectionner les individus relevant du champ des « exploitants agricoles» au sens le plus proche possible du Recensement agricole (RA). Pour ce faire, nous avons appliqué les critères utilisés par le Service de la statistique et de la prospective (SSP) dans le cadre du Bilan annuel de l'emploi agricole (BAEA, voir par exemple Agreste, 2016). Ainsi, ont été exclus les individus :

- dont le département du siège d'exploitation est renseigné comme étant Paris (75) ;

- déclarant une activité d'artisan rural ou dont le code Atexa (catégorie de risque lié aux accidents du travail couverts par la MSA) ou le code NAF (Nomenclature des Activités Françaises de l'INSEE) ne sont pas agricoles au sens du RA ${ }^{1}$;

- déclarant une activité agricole exclusivement connexe ${ }^{2}$;

- qui relèvent du régime dérogatoire de la MSA bien que n'étant pas cotisants solidaires ${ }^{3}$.

Une fois cette sélection réalisée, les données individuelles ont été consolidées à l'échelle des exploitations grâce à un identifiant unique construit à partir du numéro d'entreprise attaché à l'individu (qui correspond le plus souvent au SIRET), du numéro d'exploitation lorsque le numéro d'entreprise n'était pas renseigné (numéro attribué par la caisse MSA d'affiliation), ou enfin d'un identifiant crypté caractéristique de l'individu lui-même lorsque les deux précédents n'étaient pas renseignés. 
4 Les modifications suivantes ont ensuite été réalisées dans quelques cas d'exploitations ayant plusieurs associés: lorsque la localisation du siège renseignée différait entre associés, nous avons attribué la commune de l'associé ayant les revenus cadastraux les plus élevés; lorsque le statut juridique renseigné différait entre associés, nous avons accordé le statut le plus fréquent, en gardant la forme « la plus sociétaire $»^{4}$ si plusieurs statuts présentaient la même fréquence.

Enfin, deux variables d'intérêt central dans l'étude ont appelé des traitements particuliers pour être déterminées à l'échelle des exploitations: la Surface agricole utile (SAU) totale de l'exploitation et sa spécialisation productive :

- concernant la SAU, une simple addition des surfaces renseignées pour les individus rattachés à une exploitation ne peut suffire pour déterminer la surface totale de l'exploitation. En effet, dans la base, la surface attribuée à chaque associé est proportionnelle à la part statutaire des bénéfices qu'il détient. Lorsque la somme de ces parts était inférieure à $100 \%$, les surfaces individuelles ont été pondérées par cette somme afin de tenir compte des situations où une fraction des parts est détenue par des associés ne cotisant pas à la MSA. Lorsque la somme des parts statutaires était supérieure à $100 \%$, les surfaces n'ont pas été pondérées car il s'agissait soit d'un problème d'arrondi (la somme des parts étant alors seulement très peu supérieure à $100 \%$ ), soit de cas d'individus enregistrés sous le statut de "pluralité d'exploitation ", c'est-à-dire d'individus engagés dans plusieurs exploitations simultanément bien qu'enregistrés dans la base pour une seule. Dans de tels cas, ne disposant pas de l'information détaillée sur l'ensemble des exploitations sous-jacentes, il a été jugé préférable de conserver, sans la pondérer, l'information correspondant à l'exploitation " principale », c'est-à-dire celle pour laquelle la base est renseignée ;

7 - concernant la spécialisation productive, COTNS ne renseigne pas directement sur l'orientation technico-économique (OTEX) de l'exploitation, notion attachée aux enquêtes statistiques du SSP. Une information proche peut cependant être construite à partir de la codification Atexa, mais des hypothèses sont nécessaires pour passer des individus à l'exploitation lorsque, là encore, plusieurs associés sont présents. Plusieurs codes Atexa peuvent en effet coexister au sein d'une même exploitation si les associés cotisent pour des risques différents. Ceci peut être le cas lorsque, par exemple, l'exploitation est composée de plusieurs ateliers auxquels les individus sont chacun rattachés de façon spécifique. Mais attribuer systématiquement une orientation « mixte » dès lors que plusieurs codes Atexa sont en présence conduirait à surestimer le nombre d'exploitations mixtes. Nous avons donc procédé en trois étapes. Tout d'abord, nous avons recodé les 18 codes Atexa agricoles en s'inspirant de la nomenclature des OTEX « diffusion détaillée » et "diffusion agrégée » utilisée par le SSP ${ }^{5}$, aboutissant à une classification en 11 orientations productives («OTEF » dans la suite, $c f$. annexe du texte principal). Nous avons alors calculé le nombre d'associés par OTEF au sein de chaque exploitation et, enfin, attribué à l'exploitation l'OTEF représentant la majorité qualifiée des deux tiers des associés, l'OTEF «mixte» (9000) étant attribuée lorsque cette majorité n'était pas atteinte. 


\section{B. Comparaison avec le RA 2010}

8 Le tableau B.1 permet de comparer les nombres d'exploitations obtenus pour l'année 2010 à partir de la base COTNS et des prétraitements présentés à la section $\mathrm{A}$ avec ceux observés dans le RA, selon différents critères.

9 La colonne (e) du tableau B.1 montre que le nombre total d'exploitations obtenu à partir de COTNS est globalement sous-estimé d'environ $8 \%$ par rapport au RA. Si la non concordance exacte des deux bases n'est a priori pas surprenante tant celles-ci couvrent des champs différents et sont issues de chaînes de traitement différentes, deux éléments peuvent expliquer la sous-estimation globale de COTNS par rapport au RA. En premier lieu, par définition, la base COTNS n'enregistre ni les très petites exploitations non tenues de s'affilier à la MSA, ni les exploitations sociétaires composées uniquement de salariés, alors que celles-ci sont couvertes par le RA. En second lieu, il n'a pas été possible, comme expliqué à la section précédente, de traiter le cas des individus enregistrés avec le statut de "pluralité d'exploitation » dans COTNS, ce qui conduit sans doute, mécaniquement, à sous-estimer le nombre d'exploitations. ${ }^{6}$

Malgré cette sous-estimation globale, les données de la colonne (e) montrent également que parfois, pour tel ou tel critère, COTNS conduit au contraire à une surestimation des effectifs. Cette fois, ceci peut être dû aux hypothèses nécessaires pour consolider la base des individus à l'échelle des exploitations, en particulier en matière de détermination de l'orientation productive. Il n'en demeure pas moins que les colonnes (b) et (d) montrent finalement que, malgré ces sous et surestimations ponctuelles, COTNS permet d'arriver à des répartitions relatives des effectifs très satisfaisantes car extrêmement proches de celles observées dans le RA, quel que soit le critère choisi (taille, statut juridique et orientation productive).

11 Le lecteur intéressé trouvera chez Piet et Saint-Cyr (2016) des éléments complémentaires de comparaison à la fois à l'échelle départementale et en termes de surface totale utilisée.

Tableau A.1. Comparaison des nombres d'exploitations observés dans le RA et dérivés de COTNS pour 2010

\begin{tabular}{|l|l|l|l|l|l|}
\hline & \multicolumn{3}{|l|}{ RA } & \multicolumn{2}{l}{ COTNS } \\
\cline { 1 - 5 } & Effectif & soit en \% & Effectif & soit en \% & \\
\hline & (a) & (b) & (c) & (d) & $($ e) \\
\hline Ensemble & 489977 & $100 \%$ & 449430 & $100 \%$ & $92 \%$ \\
\hline $0 \leq$ SAU < 20 ha & 211406 & $43 \%$ & 187130 & $42 \%$ & $89 \%$ \\
\hline $20 \leq$ SAU < 50 ha & 87915 & $18 \%$ & 88904 & $20 \%$ & $101 \%$ \\
\hline $50 \leq$ SAU < 100 ha & 97440 & $20 \%$ & 93274 & $21 \%$ & $96 \%$ \\
\hline $100 \leq$ SAU < 200 ha & 72622 & $15 \%$ & 62477 & $14 \%$ & $86 \%$ \\
\hline SAU $\geq 200$ ha & 20594 & $4 \%$ & 17645 & $4 \%$ & $86 \%$ \\
\hline
\end{tabular}




\begin{tabular}{|l|l|l|l|l|l|}
\hline Individuels & 339872 & $69 \%$ & 306202 & $68 \%$ & $90 \%$ \\
\hline Sociétaires & 150105 & $31 \%$ & 143228 & $32 \%$ & $95 \%$ \\
\hline OTEF 1516 & 118763 & $24 \%$ & 104836 & $23 \%$ & $88 \%$ \\
\hline OTEF 2829 & 14568 & $3 \%$ & 16549 & $4 \%$ & $114 \%$ \\
\hline OTEF 3500 & 69955 & $14 \%$ & 59827 & $13 \%$ & $86 \%$ \\
\hline OTEF 3900 & 18599 & $4 \%$ & 13416 & $3 \%$ & $72 \%$ \\
\hline OTEF 4500 & 50226 & $10 \%$ & 57543 & $13 \%$ & $115 \%$ \\
\hline OTEF 4600 & 59530 & $12 \%$ & 58623 & $13 \%$ & $98 \%$ \\
\hline OTEF 4700 & 10793 & $2 \%$ & 12619 & $3 \%$ & $117 \%$ \\
\hline OTEF 4813 & 30536 & $6 \%$ & 21782 & $5 \%$ & $71 \%$ \\
\hline OTEF 5100 & 6277 & $1 \%$ & 5773 & $1 \%$ & $92 \%$ \\
\hline OTEF 5200 & 14809 & $3 \%$ & 10596 & $2 \%$ & $72 \%$ \\
\hline OTEF 9000 & 95921 & $20 \%$ & 87866 & $20 \%$ & $92 \%$ \\
\hline
\end{tabular}

\section{Présentation formelle du modèle mobile-stable}

12 Formellement, le modèle mobile-stable consiste à considérer une population de $N$ exploitations groupées en $J$ catégories selon leur taille. Le vecteur $n_{t+1}=\left\{n_{i, t+1}\right\}$ des effectifs par catégories à la date $t+1$ est alors donné par :

$$
\mathrm{n}_{\mathrm{t}+1}=\mathrm{n}_{t}(\mathrm{SI}+(\mathrm{I}-\mathrm{S}) \mathrm{M})(1)
$$

13 où $n_{t}=\left\{n_{i, t}\right\}$ est le vecteur des effectifs par catégories à la date $t, \mathrm{~S}$ est une matrice diagonale de dimension $J \times J$ regroupant les proportions de stables pour les différentes catégories de taille, $\mathrm{M}$ est la matrice carrée de dimension $J \times J$ décrivant le processus markovien stationnaire d'ordre 1 suivi par les «mobiles ", et est la matrice unité de dimension $J \times J .^{7}$ En pratique, l'ajout d'une catégorie supplémentaire pour modéliser les entrées et les sorties nécessite d'adapter l'équation (1) pour tenir compte du fait que, en plus de rester dans la même catégorie d'une année sur l'autre, les stables peuvent sortir et entrer entre deux années consécutives. Dans ce cas, le processus de transition caractéristique des stables n'est plus représenté par la matrice unité I mais par une matrice dont seules la diagonale principale (correspondant aux probabilités de rester dans la catégorie initiale), la dernière colonne (correspondant aux probabilités de 
sortie) et la dernière ligne (correspondant aux probabilités d'entrée) sont potentiellement non nulles.

La projection de la population à un horizon $T$ donné à partir de la dernière observation $t_{0}$ s'obtient alors en élevant chaque matrice de transition à la puissance $T-t_{0}$. Ainsi, avec la formulation du modèle mobile-stable de l'équation (3), le vecteur $\mathrm{n}_{T}$ des effectifs par catégories de taille à l'horizon $T$ est obtenu à partir des effectifs observés en $t_{0}$ comme :

$$
\mathrm{N}_{T}=\mathrm{n}_{\mathrm{t} 0}\left(\mathrm{SI}+(\mathrm{I}-\mathrm{S}) \mathrm{M}^{T-t 0}\right)(2)
$$

Enfin, pour réaliser les projections présentées ici, nous avons mis en œuvre le modèle mobile-stable dans sa version dite "en information parfaite »: au lieu d'estimer de façon endogène le type auquel appartient chaque exploitation, nous avons considéré qu'une exploitation est de type mobile dès lors qu'elle change au moins une fois de catégorie de taille, de statut juridique ou d'orientation productive sur la période d'observation, et qu'elle est de type stable si, a contrario, nous n'observons aucun de ces changements sur la période d'observation. En toute rigueur, les données disponibles ne permettant pas, en général, de suivre les exploitations sur l'ensemble de leur cycle de vie, nous nous trouvons plutôt « en information imparfaite » en ce sens que le type d'un individu n'est en réalité pas directement observable. En effet, une exploitation qui ne connaît aucun changement sur la période d'observation peut être un mobile qui n'a pas encore réalisé de transition et non pas un stable, ce dont seule une estimation économétrique comme celle utilisée par Saint-Cyr et Piet (2016) permet de tenir compte. Nous pouvons néanmoins déduire du travail de ces mêmes auteurs qu'avec 12 années de données COTNS (de 2003 à 2014) nous disposons d'une période d'observation suffisamment longue pour que l'estimation en information parfaite puisse être considérée comme robuste.

\section{Stratégie empirique de réalisation des projections}

Les projections présentées ont été réalisées en utilisant conjointement les données de la base COTNS et celles du RA 2010, ce qui permet de bénéficier des avantages des deux sources. En effet, les données annuelles à l'échelle des exploitations individuelles dérivées de COTNS permettent d'obtenir une image précise des dynamiques à l'œuvre, justifiant leur utilisation pour estimer les matrices de transition du modèle mobilestable. En revanche, la comparaison avec les données du RA montre que les données COTNS conduisent à sous-estimer d'environ $8 \%$ le nombre des structures relevant du champ agricole (voir section B). C'est pourquoi, comme le suggère Villaume (2009) et comme le fait le SSP dans l'établissement du BAEA (Agreste, 2016), nous avons utilisé les données du RA 2010 comme mesure du stock auquel ont été appliqués les flux obtenus à partir de COTNS. On aboutit ainsi à des effectifs projetés à l'horizon 2025 qui peuvent être directement rapportés aux données du RA 2010 pour mesurer les évolutions à moyen-long terme.

17 En théorie, des matrices de probabilités de transition peuvent être calculées à partir de la base COTNS à différentes échelles (nationale, régionale ou départementale) et selon les différents critères retenus (toutes exploitations confondues, par statuts juridiques et par orientations productives). En pratique, les effectifs sur lesquels les calculs 
s'appuient doivent être suffisants pour que les matrices obtenues soient dites stables et conduisent à des projections robustes (Berchtold, 1998). Si l'on retient le seuil d'au minimum 30 observations dans une des classes de taille initiale pour pouvoir calculer une matrice de transition donnée, il s'avère que les six matrices d'intérêt, correspondantes aux trois critères (toutes exploitations confondues, par statuts juridiques et par orientations productives) pour chacun des deux types d'individus (mobiles et stables), peuvent toutes être calculées à l'échelle nationale. A contrario, aux échelles régionale et départementale, certaines matrices ne doivent pas être calculées, faute d'effectifs suffisants dans telle ou telle classe de taille et pour tel ou tel type d'exploitations. En conséquence, l'objectif du travail étant de fournir des projections cohérentes et exhaustives à l'échelle de la France entière, ce sont les matrices nationales qui ont été appliquées aux effectifs départementaux. Ceci revient à faire l'hypothèse que les exploitations se comportent de façon similaire quel que soit le département, et donc à négliger d'éventuelles spécificités locales. Seule une étude détaillée à l'échelle de quelques départements tests, où les effectifs seraient suffisants, permettrait d'évaluer l'éventuel biais ainsi introduit. Cependant, il n'y a pas de raison de penser qu'un tel biais soit systématiquement à la hausse ou à la baisse puisque la matrice nationale constitue, par définition, une matrice " moyenne». Il n'en demeure pas moins que les résultats départementaux présentés dans le texte sont à considérer avec plus de précaution que les résultats agrégés à l'échelle nationale.

Enfin, il faut noter que les matrices obtenues selon des critères différents (toutes exploitations confondues, par statuts juridiques ou par orientations productives) sont différentes par construction, conduisant de facto à des projections différentes. Les matrices calculées "toutes exploitations confondues", basées sur les effectifs les plus importants, s'avérant les plus robustes, la projection les utilisant a été retenue comme simulation de référence. Afin de conserver une cohérence dans les résultats présentés, les projections réalisées selon les deux autres critères (par statuts juridiques ou par orientations productives) ont été ajustées grâce à une simple règle de proportionnalité afin de retomber sur les mêmes chiffres globaux.

\section{E. Ajustement du modèle aux données}

Afin de vérifier l'adéquation du modèle aux données, et en particulier valider la pertinence du modèle stationnaire, les probabilités de transition présentées à la section 4 de l'article ont été appliquées aux effectifs observés chaque année dans la base COTNS afin de simuler ceux de l'année suivante. La figure E.1 confronte ainsi les effectifs observés et estimés pour les 95 départements étudiés, les 5 classes de taille considérées et les 11 années simulées sur la période d'observation (2004 à 2014 inclus), d'une part toutes exploitations confondues (soit $95 \times 5 \times 11=5225$ points, figure E.1a), d'autre part pour les deux statuts juridiques (soit $95 \times 5 \times 11 \times 2=10450$ points, figure E.1b) et enfin pour les 11 orientations productives (soit $95 \times 5 \times 11 \times 11=57475$ points, figure E.1c).

Le modèle s'ajuste très bien aux données puisque les régressions donnent un coefficient d'ajustement supérieur à 0.99 et une erreur moyenne entre effectifs observés et simulés inférieure à $0.1 \%$ dans les trois cas. Cependant, outre quelques cas isolés dans les trois graphiques, nous constatons une plus grande dispersion lorsque l'analyse est conduite par OTEF. Le modèle stationnaire est donc légèrement moins performant dans ce cas. Finalement, la confrontation des effectifs totaux simulés et observés confirme 
l'adéquation du modèle (figure E.2), ce dernier permettant bien de reproduire l'évolution observée quel que soit le critère d'analyse retenu. 
Figure E.1. Ajustements des effectifs simulés par départements et par critères aux effectifs observés 

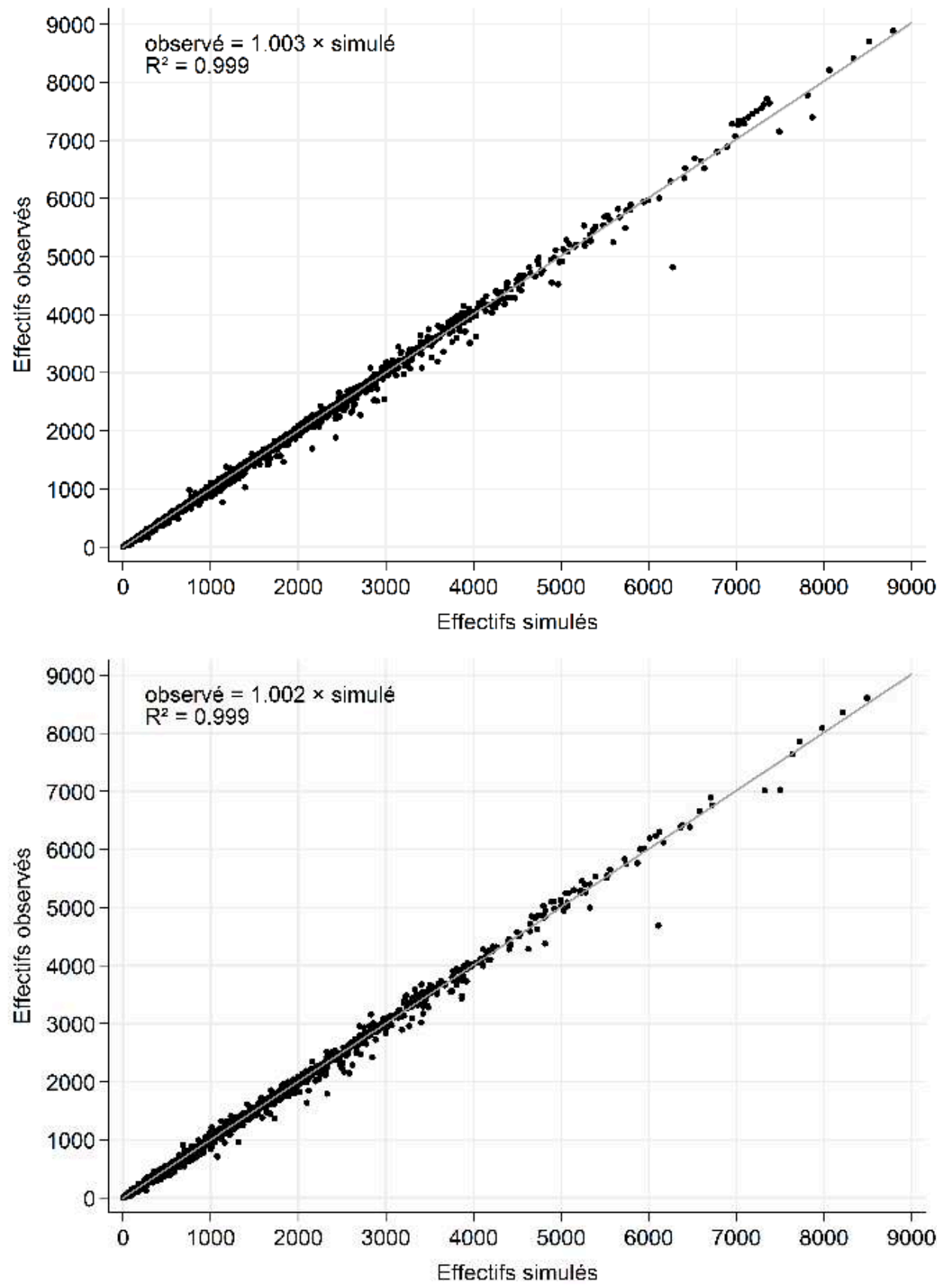


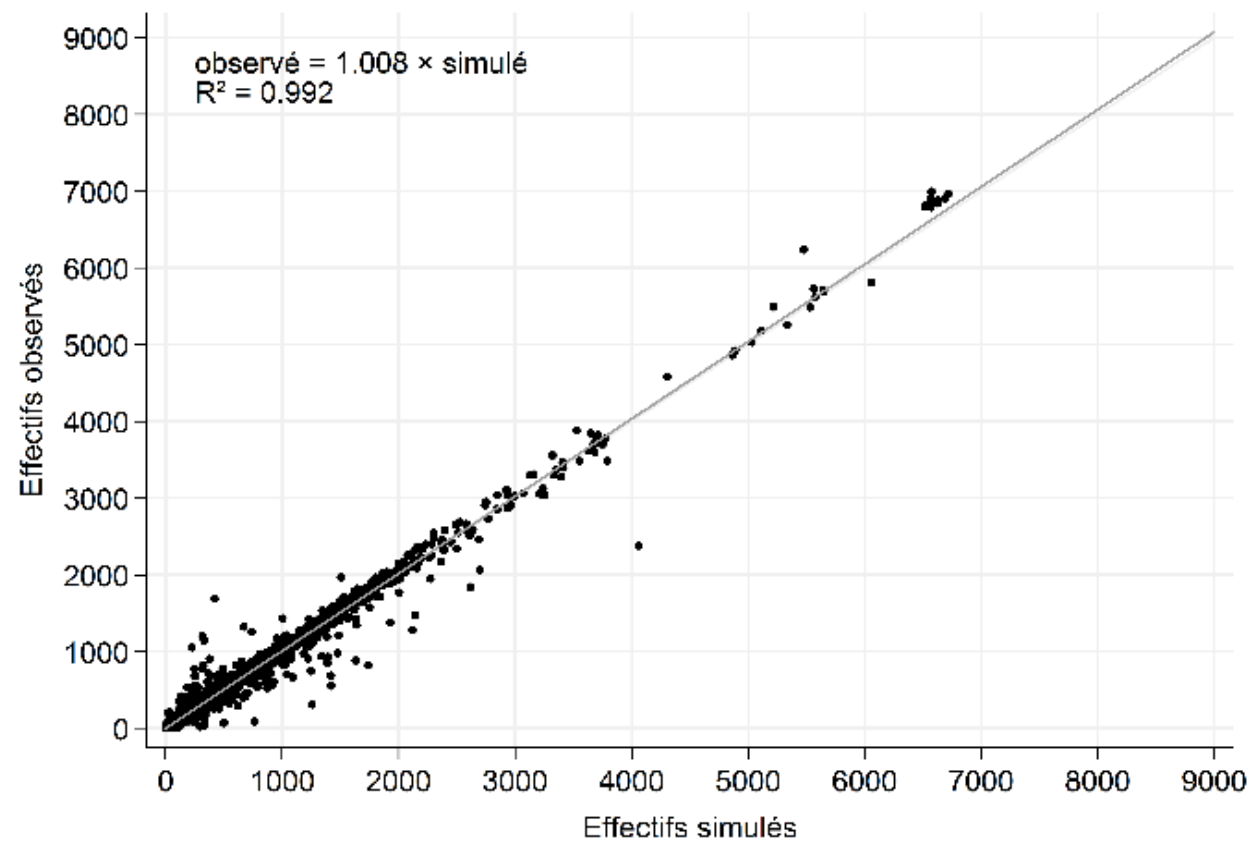

a) Toutes exploitations confondues (5 225 points) b) Par statuts juridiques (10 450 points) c) Par orientations productives (57 475 points)

Source : COTNS 2003-2014 (MSA), traitement des auteurs. 
Figure E.2. Ajustements des effectifs totaux simulés aux effectifs totaux observés 

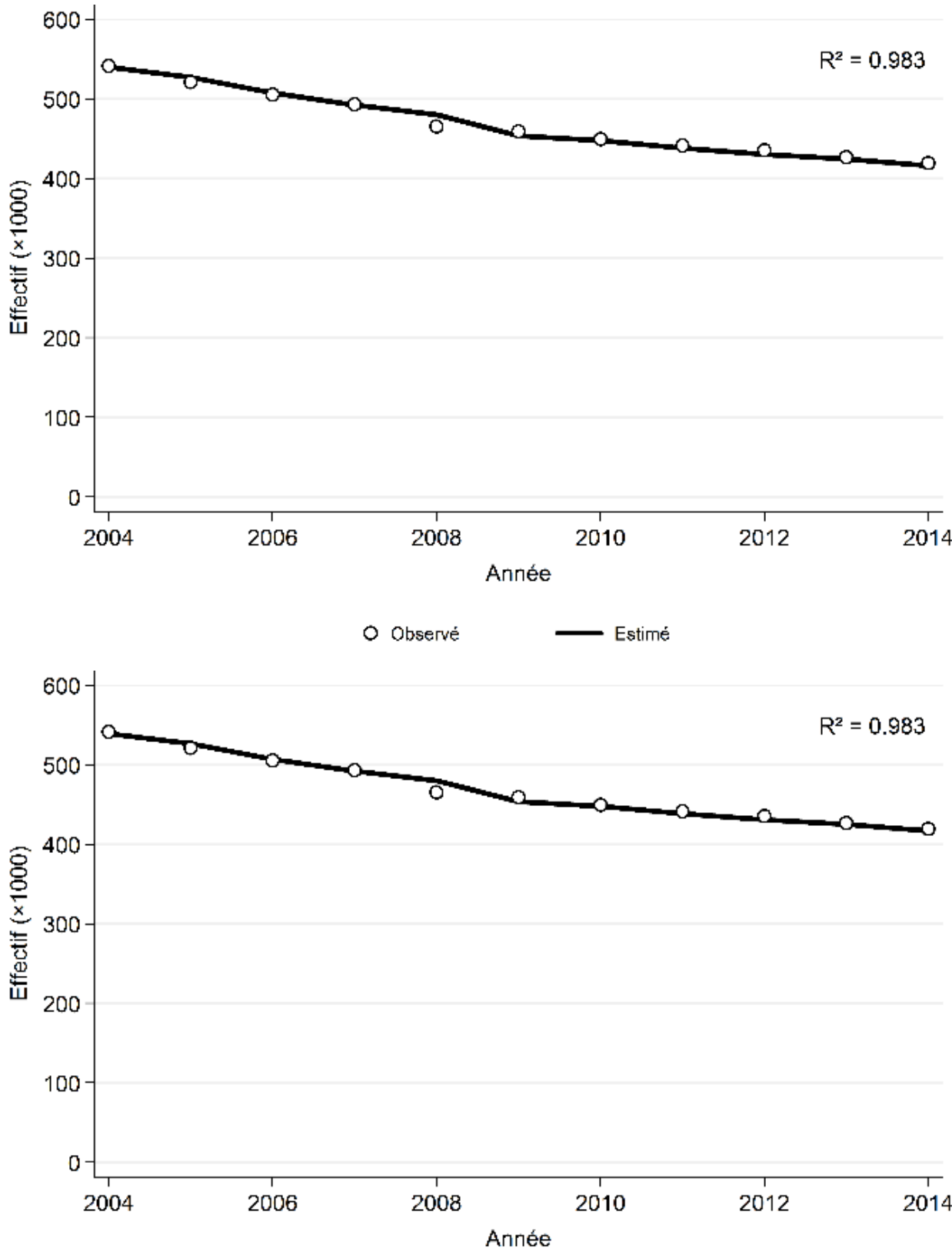

O Observé $\quad$ Estimé 


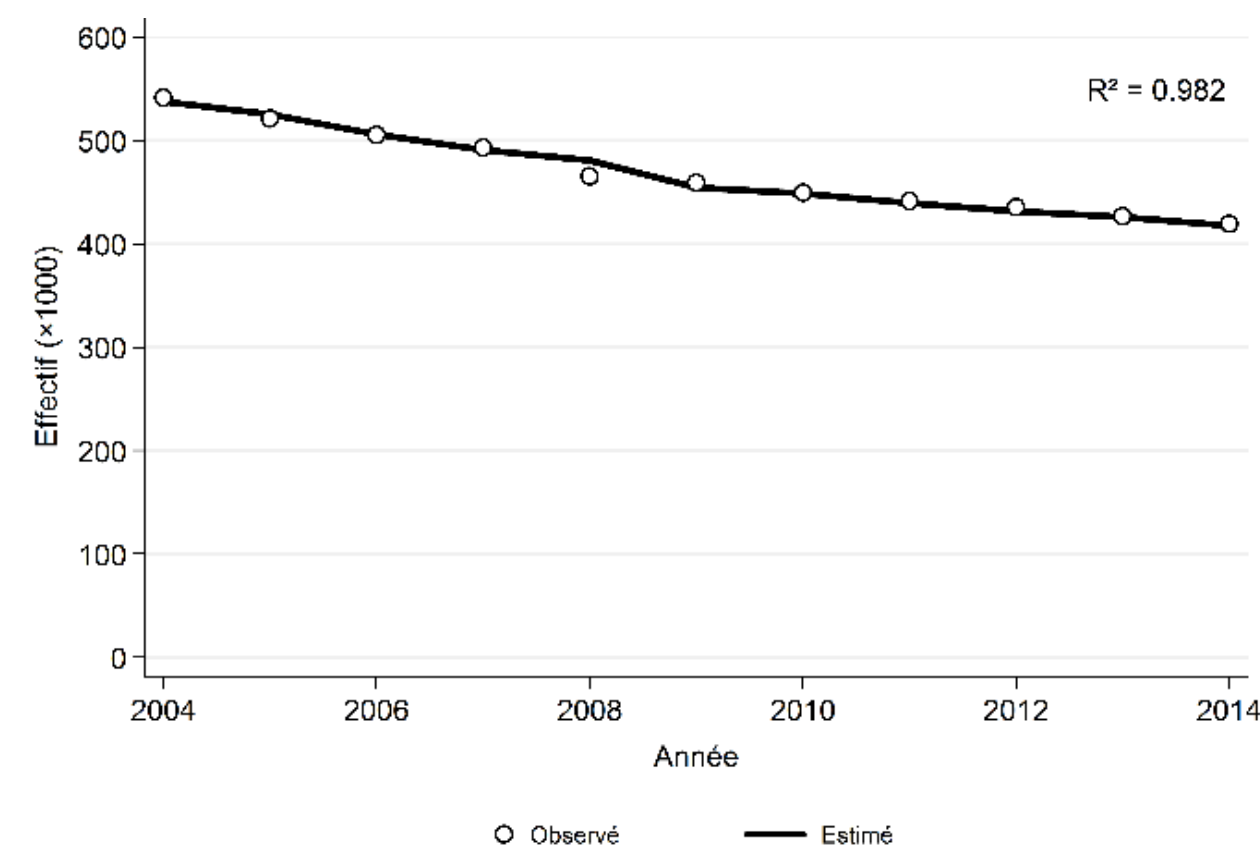

a) Toutes exploitations confondues b) Par statuts juridiques c) Par orientations productives Source : COTNS 2003-2014 (MSA), traitement des auteurs.

\section{BIBLIOGRAPHIE}

Agreste (2016). Le bilan annuel de l'emploi agricole. Résultats 2014 et estimations 2015. Agreste Chiffres et Données Agriculture, n²34, 71 p., available at http://www.lafranceagricole.fr/r/Publie/ FA/p1/Infographies/Web/2016-06-30/Agreste_emploi_30062016.pdf.

Berchtold A. (1998). Chaînes de Markov et modèles de transition : application aux sciences sociales. Éditions Hermès, 284 p.

Piet L., Saint-Cyr L. D. F. (2016). Projection de la population des exploitations agricoles françaises à l'horizon 2025. Working Paper SMART-LERECO, n¹6-11, 35 p.

Saint-Cyr L. D. F., Piet L. (2016). Movers and stayers in the farming sector: accounting for unobserved heterogeneity in structural change. Journal of the Royal Statistical Society, Series C Applied Statistics, 19 p., available at http://onlinelibrary.wiley.com/doi/10.1111/rssc.12196/pdf.

Villaume (2009). Emploi agricole : recensement et comparaison des sources. Exemple : rapprochement de l'enquête structure des exploitations et du fichier MSA non salariés. Document de travail E 2009/08 de la Direction des Statistiques d'Entreprises, INSEE, 59 p.

\section{NOTES}

1. Pour l'Atexa, il s'agit des codes 6 (sylviculture), 18 (conchyliculture), 20 (marais salants), 21 (exploitation de bois), 22 (scieries fixes), 23 (entreprises de travaux agricoles), 24 (entreprises de 
jardins, paysagistes, de reboisement), 25 (mandataires des sociétés ou caisses locales d'assurances mutuelles agricoles) et 26 (artisans ruraux). Pour la NAF, il s'agit, avant 2008, des codes 927C, 050A, 050C, 014B, 804C, 926C, 927C, 930N et 950Z, et, après 2008, des codes 0162Z, 0311Z, 0312Z, 0321Z, 0322Z, 8130Z, 8551Z, 9319Z, 9329Z, $9609 \mathrm{Z}$ et $9700 \mathrm{Z}$.

2. À savoir les individus dont l'activité est définie uniquement en fonction d'un nombre d'heures de travail et non pas du critère "normal » d'assujettissement, c'est-à-dire en référence à la Surface minimale d'installation (SMI) avant la mise en œuvre de la loi d'avenir pour l'agriculture de 2014, et à l'Activité minimale d'assujettissement (AMA) depuis.

3. Un affilié est identifié comme " cotisant solidaire ", lorsque l'importance de l'activité agricole ne permet pas une affiliation en qualité de chef d'exploitation ou d'entreprise agricole; avant la mise en œuvre de la loi d'avenir pour l'agriculture de 2014, c'était par exemple le cas des exploitations représentant moins d'une demi-SMI.

4. Par exemple : entre individuel et GAEC on a gardé GAEC ; entre GAEC et EARL on a gardé EARL ; entre EARL et SCEA on a gardé SCEA ; etc.

5. Cf. le document http://www.agreste.agriculture.gouv.fr/IMG/pdf/nomenlature-Otex.pdf sur le site internet Agreste.

6. Avec les traitements de consolidation présentés dans l'article, 20305 exploitations sont classées en "pluralité d'exploitation» en 2010, soit environ 4,5\% des 449430 exploitations obtenues.

7. Avec ce formalisme, le modèle de chaîne de Markov homogène est obtenu en considérant que toutes les exploitations suivent un seul et unique processus stochastique markovien, c'est-à-dire que les proportions de stables sont toutes nulles.

\section{AUTEUR}

\section{LEGRAND DUNOLD FILS SAINT-CYR}

Smart-Lereco, Agrocampus Ouest, Inra, Rennes ; legrand.saint-cyr@inra.fr 